OPEN ACCESS

Edited by:

Sanket J. Joshi,

Sultan Qaboos University, Oman

Reviewed by:

Abhishek Walia,

Chaudhary Sarwan Kumar Himachal Pradesh Krishi Vishvavidyalaya, India

Jagat Rathod,

National Cheng Kung University,

Taiwan

Ketan D. Patel,

University at Buffalo, United States

*Correspondence:

Hong Shen

hshen@scau.edu.cn

Specialty section:

This article was submitted to

Microbiotechnology,

a section of the journal

Frontiers in Microbiology

Received: 19 March 2021

Accepted: 14 May 2021

Published: 17 June 2021

Citation:

Cui D, Yang J, Lu B and Shen H (2021) Efficient Preparation

of Chitooligosaccharide With

a Potential Chitosanase Csn-SH and Its Application for Fungi Disease

Protection

Front. Microbiol. 12:682829. doi: 10.3389/fmicb.2021.682829

\section{Efficient Preparation of} Chitooligosaccharide With a Potential Chitosanase Csn-SH and Its Application for Fungi Disease Protection

\author{
Dandan Cui ${ }^{1}$, Jin Yang ${ }^{1}$, Bosi Lu' ${ }^{1}$ and Hong Shen ${ }^{1,2 *}$ \\ ' College of Natural Resources and Environment, South China Agricultural University, Guangzhou, China, ${ }^{2}$ Guangdong \\ Provincial Key Laboratory of Eco-Circular Agriculture, Guangzhou, China
}

Chitosanase plays a vital role in bioactive chitooligosaccharide preparation. Here, we characterized and prepared a potential GH46 family chitosanase from Bacillus atrophaeus BSS. The purified recombinant enzyme Csn-SH showed a molecular weight of $27.0 \mathrm{kDa}$. Csn-SH displayed maximal activity toward chitosan at pH 5.0 and $45^{\circ} \mathrm{C}$. Thin-layer chromatography and electrospray ionization-mass spectrometry indicated that Csn-SH mainly hydrolyzed chitosan into $(\mathrm{GlcN})_{2},(\mathrm{GlcN})_{3}$, and $(\mathrm{GlcN})_{4}$ with an endo-type cleavage pattern. Molecular docking analysis demonstrated that Csn-SH cleaved the glycoside bonds between subsites -2 and +1 of $(\mathrm{GlcN})_{6}$. Importantly, the chitosan hydrolysis rate of Csn-SH reached $80.57 \%$ within 40 min, which could reduce time and water consumption. The hydrolysates prepared with Csn$\mathrm{SH}$ exhibited a good antifungal activity against Magnaporthe oryzae and Colletotrichum higginsianum. The above results suggested that Csn-SH could be used to produce active chitooligosaccharides efficiently that are biocontrol agents applicable for safe and sustainable agricultural production.

Keywords: chitosanase, Bacillus atrophaeus, chitooligosaccharide, antifungal activity, hydrolysis rate

\section{INTRODUCTION}

Chitin is the second largest natural polysaccharide after cellulose and exists widely in arthropods exoskeletons, fungi, and insect intestinal mucosa. Because of its insolubility in common solvents, chitin is still a rarely utilized natural biomass (Luo et al., 2020). Chitosan is a partially or completely deacetylated derivative of chitin that consists mainly of D-glucosamine (GlcN) linked with $\beta-1,4$ glycosidic bonds and contains a small amount of $\mathrm{N}$-acetyl-D-glucosamine (GlcNAc) (Zheng et al., 2021). Chitosan exhibits good biodegradability and biocompatibility and is nontoxic and soluble in weak acid. Thus, it has been widely used in gene therapy, drug delivery, wound repair, and microbial growth inhibition (Yang et al., 2020). Nevertheless, chitosan is insoluble in water and soluble only in some inorganic and organic acids, such as acetic, hydrochloric, lactic, and citric acids, among which acetic acid is the most commonly used (Nascimento et al., 2020). Chitooligosaccharides (COSs), are degradation products of chitosan consisting of depolymerized derivatives of chitosan with a degree of polymerization (DP) ranging from 2 to 10 (Benchamas et al., 2021). COSs are 
nontoxic, water-soluble, and biocompatible and have been widely used as antitumor (Jiang et al., 2020), drug-delivery (Wang et al., 2021a), antimicrobial (Silva et al., 2021), antifungal (Zhou et al., 2020), immune-activating (Zhang et al., 2021), and food preservation agents (Dutta et al., 2012).

Chitosan has been shown to be degraded to COSs by chemical methods or enzymatic processes. However, chemical methods have many drawbacks, such as polluting the environment, harming the physicochemical properties of COSs, and presenting high costs. In contrast, enzymatic hydrolysis presents many advantages, such as an absence of damage to the active group structure, safety, less environmental pollution, high yields, and controllability. Many enzymes have been reported to hydrolyze chitosan, such as cellulase, papain, pepsin, pectinase, and lipase, and chitosanase (EC. 3.2.1.132) hydrolyzes chitosan specifically (Wang et al., 2021b).

According to amino acid sequence similarity, chitosanases are divided into seven families in the CAZy database, including GH3, GH5, GH7, GH8, GH46, GH75, and GH80, among which the GH46, GH75, and GH80 families only contain chitosanases (Viens et al., 2015). GH46 is currently the most extensively studied family of chitosanase and mainly consists of enzymes derived from Bacillus subtilis, Pseudomonas sp., Streptomyces sp., Kitasatospora sp., and other bacterial genera (Viens et al., 2015). Owing to the low enzymatic activities of most original strains, cloned and exogenously expressed chitosanases have attracted extensive attention of people. In recent years, the exogenous expression of chitosanase derived from Bacillus, Staphylococcus, Microbacterium, Streptomyces, and other bacterial genera has been reported, among which Streptomyces and Bacillus have provided the greatest numbers of these enzymes. However, there are few reports regarding the heterologous expression of chitinase and chitosanase from Bacillus atrophaeus.

The B. atrophaeus BSS genome sequences were submitted to the database of National Center for Biotechnology Information (NCBI) in 2014. A chitosanase gene was annotated in the genome. In the present study, the chitosanase gene csn from $B$. atrophaeus BSS was cloned and heterologously expressed in Escherichia coli BL21 (DE3). The physicochemical properties, hydrolytic characteristics, and hydrolysis rate of the recombinant chitosanase Csn-SH were investigated. Furthermore, the antifungal activities of the COSs prepared with Csn-SH were also evaluated.

\section{MATERIALS AND METHODS}

\section{Materials}

Colloidal chitosan (degree of deacetylation [DD] $\geq 85 \%$ ) and water-soluble chitosan (DD $\geq 95 \%$ ) were purchased from Qingdao BZ Oligo Biotech Co., Ltd. (Qingdao, China). $(\mathrm{GlcN})_{2-6}$ (DP2-6) were purchased from Huizhou Long Dragon Biotechnology Co., Ltd. (Huizhou, China). Colloidal chitin and carboxymethyl cellulose (CMC) were purchased from Aladdin (Shanghai, China). COSs (DP2-7) were purchased from Guangzhou SGY Agricultural Science and Technology Co., Ltd. (Guangzhou, China). Magnaporthe oryzae and Colletotrichum higginsianum were kindly provided by Prof. Yunfeng Li (College of Plant Protection) and Prof. Shujie Feng (College of Horticulture), South China Agricultural University, Guangzhou, China, respectively.

\section{Gene Cloning and Sequence Analysis}

A new isolated $B$. atrophaeus strain BSS was identified, and the genome sequences were submitted to NCBI database by H. E. Daligault et al. in 2014. Based on the genome information of B. atrophaeus BSS (GenBank: CP007640.1), a GH46 chitosanase gene $\operatorname{cs} n$ was annotated. The open reading frame was mapped by ORF finder. ${ }^{1}$ Prediction of signal peptides was performed using the SignalP 5.0. ${ }^{2}$ The analysis of conserved domain was carried out by the Conserved Domain Database of NCBI. The theoretical molecular weight (Mw) and isoelectronic point (pI) were computed using the tool of ExPASy ProtParam without the signal peptide. ${ }^{3}$ Multiple sequence alignment was performed using Clustal Omega ${ }^{4}$ and obtained using DNAMAN (9.0) and ESPript $3.0^{5}$ after the removal of signal peptide. Phylogenetic and molecular evolutionary analyses were conducted by using MEGA 7.0 software.

To explore the potential catalytic function of $\operatorname{cs} n$, we optimized the gene codon according to the $E$. coli codon preference. The optimized gene was chemically synthesized by Sangon (Shanghai, China) without signal peptide and inserted into plasmid pET-28a (+). The recombinant plasmid was transformed to E. coli BL21 (DE3) competent cells by heat shock method for expression of the chitosanase. The positive transformants were selected on Luria-Bertani (LB) agar plates supplemented with 50 $\mu \mathrm{g} / \mathrm{mL}$ kanamycin.

\section{Gene Expression and Purification}

The recombinant $E$. coli BL21 (DE3) cells were cultivated in LB medium containing $50 \mu \mathrm{g} / \mathrm{mL}$ kanamycin at $37^{\circ} \mathrm{C}$ in a shaker (180 revolutions/min). When $\mathrm{OD}_{600}$ of the culture reached 0.6 , isopropyl- $\beta$-D-thiogalactopyranoside (IPTG) was added into the culture at a final concentration of $0.01-1 \mathrm{mM}$. The induced cultures were further grown at $20,25,30$, or $35^{\circ} \mathrm{C}$ for $12 \mathrm{~h}$, and the cells were harvested by centrifugation at $10,000 \mathrm{~g}$ for $10 \mathrm{~min}$, washed twice with $20 \mathrm{mM}$ sodium phosphate buffer ( $\mathrm{pH} 7.0$ ), and stored at $-20^{\circ} \mathrm{C}$.

The harvested cells were resuspended in sodium phosphate buffer $(20 \mathrm{mM}, \mathrm{pH} 7.0)$ and disrupted by sonication, and then the debris was removed by centrifugation at $10,000 \mathrm{~g}$ for $10 \mathrm{~min}$ at $4^{\circ} \mathrm{C}$. The resulting crude extract was purified by Ni-NTA column (Sangon, China) according to the protocol, and CsnSH was collected and carried out by sodium dodecyl sulfatepolyacrylamide gel electrophoresis (SDS-PAGE). The protein concentration was determined using the Bradford method, and bovine serum albumin was used as the standard (Luo et al., 2020).

\footnotetext{
${ }^{1}$ https://www.ncbi.nlm.nih.gov/orffinder/

${ }^{2}$ http://www.cbs.dtu.dk/services/SignalP/

${ }^{3}$ https://web.expasy.org/protparam/

${ }^{4}$ http://www.ebi.ac.uk/Tools/msa/clustalo/

${ }^{5}$ http://espript.ibcp.fr/ESPript/cgi-bin/ESPript.cgi
} 


\section{Enzyme Activity Assay}

Chitosanase assays were performed according to the method (Sun et al., 2020). Briefly, $100 \mu \mathrm{L}$ properly diluted enzyme solution was mixed with $900 \mu \mathrm{L}$ of $0.5 \%$ (wt/vol) colloidal chitosan, and then $1 \mathrm{~mL}$ sodium acetate buffer $(20 \mathrm{mM}, \mathrm{pH}$ 5.0) was added to the above mixture. The reaction mixture was incubated at $40^{\circ} \mathrm{C}$ for $10 \mathrm{~min}$. The reaction was quenched by adding $1 \mathrm{~mL}$ dinitrosalicylic acid solution and then incubated in boiling water for $10 \mathrm{~min}$. The reducing sugar in the supernatant was determined by measuring absorbance at $520 \mathrm{~nm}$ with Dglucosamine as the standard. All the experiments were performed in triplicate. One unit $(\mathrm{U})$ of chitosanase activity was defined as the amount of enzyme needed to liberate $1 \mu \mathrm{mol} \mathrm{D}$ glucosamine-equivalent-reducing sugar per minute in the above assay conditions.

\section{Characterization of the Purified Csn-SH}

Colloidal chitosan $(0.5 \%, \mathrm{wt} / \mathrm{vol})$ was used as the substrate to determine the enzymatic characterization of Csn-SH. The optimal $\mathrm{pH}$ for Csn-SH activity was determined by measuring the enzyme activity from $\mathrm{pH} 2.5$ to 6.0 using $20 \mathrm{mM}$ various buffers as follows: Gly-HCl buffer, $\mathrm{pH} 2.5$ to 3.5; sodium acetate buffer, $\mathrm{pH} 3.5$ to 6.0. The reaction mixture consisted of $100 \mu \mathrm{L}$ CsnSH, $900 \mu \mathrm{L}$ chitosan, and $1 \mathrm{~mL}$ buffer. To determine $\mathrm{pH}$ stability, $100 \mu \mathrm{L}$ Csn-SH was added to $900 \mu \mathrm{L}$ different $20 \mathrm{mM}$ buffers without substrate at $45^{\circ} \mathrm{C}$ for $1 \mathrm{~h}$. The buffers used were as follows: Gly-HCl buffer, $\mathrm{pH}$ 2.5-3.5; sodium acetate buffer, $\mathrm{pH}$ 3.5-6.0; sodium phosphate buffer, $\mathrm{pH} 6.0-8.0$; and Tris- $\mathrm{HCl}$ buffer, $\mathrm{pH}$ 8.0-10.0. After preincubation, the residual chitosanase activity was measured under standard assay conditions.

The optimal temperature for Csn-SH activity was examined by incubating the enzyme with chitosan at $20^{\circ} \mathrm{C}$ to $80^{\circ} \mathrm{C}$ in $20 \mathrm{mM}$ sodium acetate buffer ( $\mathrm{pH}$ 5.0). The enzymatic thermostability was evaluated by measuring the residual activity of Csn-SH incubated at temperature ranging from $30^{\circ} \mathrm{C}$ to $60^{\circ} \mathrm{C}$ in $20 \mathrm{mM}$ sodium acetate buffer ( $\mathrm{pH}$ 5.0) for 0.5 to $2 \mathrm{~h}$. The residual enzymatic activity was tested in standard assay conditions.

The effects of metal cations $\left(\mathrm{NaCl}, \mathrm{KCl}, \mathrm{Li}_{2} \mathrm{SO}_{4}, \mathrm{MgCl}_{2}, \mathrm{CaCl}_{2}\right.$, $\mathrm{CuCl}_{2}, \mathrm{MnCl}_{2}, \mathrm{CoCl}_{2}, \mathrm{ZnSO}_{4}, \mathrm{FeSO}_{4}, \mathrm{PbSO}_{4}, \mathrm{FeCl}_{3}$, and $\mathrm{AlCl}_{3}$ ) and chemical (EDTA) on the enzymatic activity of Csn-SH were determined at the final concentration of 1 and $5 \mathrm{mM}$ in the reaction mixture at $45^{\circ} \mathrm{C}$ for $10 \mathrm{~min}$. The catalytic activity without any chemicals was used as the control, and the activity was defined as $100 \%$.

\section{Substrate Specificity}

Substrate specificity of Csn-SH was determined in $20 \mathrm{mM}$ sodium acetate buffer ( $\mathrm{pH} \mathrm{5.0)}$ at $45^{\circ} \mathrm{C}$ for $10 \mathrm{~min}$. The tested substrates $(0.5 \% \mathrm{wt} / \mathrm{vol})$ included colloidal chitosan ( $\mathrm{DD} \geq 85 \%)$, watersoluble chitosan (DD $\geq 95 \%)$, colloidal chitin, and CMC.

\section{Determination of the Kinetic Parameters of Csn-SH}

The kinetic parameters of Csn-SH were analyzed under optimal conditions as described previously and used various concentrations of colloidal chitosan. [S] indicated the chitosan concentrations, which ranged from 0.2 to $2.0 \mathrm{mg} / \mathrm{mL}$ with an interval of 0.2 . The reactions were performed at $45^{\circ} \mathrm{C}$ for $10 \mathrm{~min}$ in sodium acetate buffer ( $20 \mathrm{mM}, \mathrm{pH} 5.0)$. The $K_{m}$ and $V_{\max }$ values were calculated by the Lineweaver-Burk equation (Zhou et al., 2020).

\section{Hydrolysis Pattern of Csn-SH}

The hydrolysis properties of Csn-SH were investigated by using colloidal chitosan and COSs viz. $(\mathrm{GlcN})_{2},(\mathrm{GlcN})_{3},(\mathrm{GlcN})_{4}$, $(\mathrm{GlcN})_{5}$, and $(\mathrm{GlcN})_{6}$ as substrates. The hydrolysates prepared with Csn-SH were analyzed through thin-layer chromatography (TLC) and electrospray ionization-mass spectrometry (ESI-MS). In brief, $100 \mu \mathrm{L}$ Csn-SH $(20 \mathrm{U} / \mathrm{mL})$ was mixed with $900 \mu \mathrm{L}$ $1 \%(\mathrm{wt} / \mathrm{vol})$ different substrates including $(\mathrm{GlcN})_{2},(\mathrm{GlcN})_{3}$, $(\mathrm{GlcN})_{4},(\mathrm{GlcN})_{5},(\mathrm{GlcN})_{6}$, and colloidal chitosan, respectively, and $1 \mathrm{~mL}$ sodium acetate buffer $(20 \mathrm{mM}, \mathrm{pH} 5.0)$ was added into the mixture and incubated at $45^{\circ} \mathrm{C}$. Samples were withdrawn at different times and immediately boiled for $10 \mathrm{~min}$ before centrifuged at $12,000 \mathrm{~g}$ for $5 \mathrm{~min}$. The supernatant samples were spotted in a TLC plate (Silica gel 60 F254 aluminum sheet; Merck, Darmstadt, Germany), developed in isopropanol/ammonium $(2: 1, \mathrm{vol} / \mathrm{vol})$ solvent, and sprayed with $0.1 \%$ ninhydrin reagent (dissolved in ethanol). The hydrolysates were visualized by heating the plate at $110^{\circ} \mathrm{C}$ in an oven for $5 \mathrm{~min}$. ESI-MS studies were performed in positive-ion mode with a ratio of mass to charge in the range of 50 to $2,000(\mathrm{~m} / \mathrm{z})$. The scan mode was used under a capillary needle at $3.5 \mathrm{kV}$, and the ion source temperature was kept at $180^{\circ} \mathrm{C}$.

\section{Homology Modeling and Molecular Docking}

Homology models of Csn-SH were generated using the Modeller 9.19 with the chitosanase from B. subtilis MY002 (PDB: 7C6C; identity: 91.3\%) as the template ( $\mathrm{Li}$ et al., 2021). Molecular docking between $\mathrm{Csn}-\mathrm{SH}$ and $(\mathrm{GlcN})_{6}$ was performed by Autodock (Lyu et al., 2014). Then, the Csn-SH complexed with $(\mathrm{GlcN})_{6}$ was superimposed on the structure of chitosanase CsnMY002 from B. subtilis MY002 (PDB: 7C6C) using PyMOL.

\section{Inhibitory Effects of COSs on Fungi}

The inhibitory effects of COSs prepared with Csn-SH on the phytopathogenic fungi $M$. oryzae and C. higginsianum were investigated. The fungi preserved on an inclined surface were inoculated onto the potato dextrose agar (PDA) plate and cultured at $28^{\circ} \mathrm{C}$ for 3 days to obtain a fresh colony. Then, a sterile blade was used to cut the edge of the colony to obtain the fungus cakes with a diameter of $6 \mathrm{~mm}$. The mycelial side of the cake was inoculated down to the center of PDA plates separately containing $0.1,0.5,1.0,2.0,4.0$, and $8.0 \mathrm{mg} / \mathrm{mL}$ COSs. COSs-Csn indicated the COSs prepared with Csn-SH, whereas COSs-SGY represented COSs purchased from Guangzhou SGY Agricultural Science and Technology Co., Ltd. For fungi M. oryzae, the plates were cultured at $28^{\circ} \mathrm{C}$ for 6 days, and the plates of C. higginsianum were cultured at $25^{\circ} \mathrm{C}$ for 7 days. The PDA plate without COSs was used as the control. Finally, the diameters of the fungal mycelia were measured. The growth 
inhibition rate was calculated according to the previous study (Zhou et al., 2020).

\section{RESULTS AND DISCUSSION}

\section{Sequence Analysis of Csn-SH}

The csn gene from B. atrophaeus strain BSS genome (GenBank: CP007640.1) contained an 825-bp open reading frame, which encoded a chitosanase (Csn-SH) of 274 amino acids. A 32amino-acid signal peptide was predicted at the $\mathrm{N}$-terminus via SignalP analysis. Domain structure prediction analysis indicated that Csn-SH was a single-domain protein with a GH family 46 catalytic regions between residues 38 and 261 . The theoretical $\mathrm{Mw}$ and $\mathrm{pI}$ of Csn-SH were $27.4 \mathrm{kDa}$ and 5.62 without signal peptide, respectively.

According to the sequence alignments and phylogenetic tree analysis, the chitosanases of GH46 family were grouped into five clusters $\mathrm{A}, \mathrm{B}, \mathrm{C}, \mathrm{D}$, and $\mathrm{E}$, and almost all chitosanases in cluster B were derived from Bacilli (Viens et al., 2015). The phylogenetic tree analysis results indicated that $\mathrm{Csn}-\mathrm{SH}$ was a novel member of cluster B (Figure 1A) and showed higher homology with the chitosanase from Bacillus amyloliquefaciens MJ-1 (Hong and Kang, 2006). Glutamic acid (E) and aspartic acid (D) are two conserved catalytic residues in the GH46 family (Takasuka et al., 2014); the corresponding residues of Csn-SH were E19 and D35 (Figure 1B, labeled with yellow circles). Figure 1B also showed that multiple substrate-binding sites (labeled with hollow squares) played important roles in the substrate preference of Csn-SH (Yang et al., 2019).

\section{Expression and Purification of Csn-SH}

The $c s n$ gene was cloned into the pET-28( $\left.\mathrm{a}^{+}\right)$vector with a C-terminal His tag and transferred into E. coli BL21 (DE3). Then, the recombinant protein was induced by different IPTG concentrations at temperatures ranging from $20^{\circ} \mathrm{C}$ to $35^{\circ} \mathrm{C}$. The results indicated that $\mathrm{Csn}-\mathrm{SH}$ showed its maximum enzymatic activity in the presence of $0.1 \mathrm{mmol} / \mathrm{L}$ IPTG at $20^{\circ} \mathrm{C}$ and 0.05 $\mathrm{mmol} / \mathrm{L}$ IPTG at $30^{\circ} \mathrm{C}$, respectively (Figure $1 \mathrm{C}$ ). Because the IPTG is a potentially toxic chemical for cell growth (Zheng et al., 2021), the condition of $0.05 \mathrm{mmol} / \mathrm{L}$ IPTG at $30^{\circ} \mathrm{C}$ was chosen for further study. After purification in a Ni-NTA column, the enzymatic activity of Csn-SH increased from 29.04 to $366.14 \mathrm{U} / \mathrm{mg}$. Compared with other chitosanases of the GH46 family heterologously expressed in E. coli BL21 (DE3), the enzymatic activity of Csn-SH was close to that of CsnQ from Bacillus sp. Q1098 (371.6 U/mg) (Ma et al., 2020) and much higher than that of Csn-BAC from Bacillus sp. MD-5 (41.67 U/mg) (Yang et al., 2020) and lower than CsnS from Serratia sp. QD07 (426.7 U/mg) after 60-h fermentation (Zheng et al., 2021). This indicated that the enzymatic activity of Csn-SH might be much higher after high density fermentation.

SDS-PAGE results showed that the Mw of purified CsnSH was approximately $27.0 \mathrm{kDa}$ (Figure 1D), which was in accordance with the theoretical $\mathrm{Mw}(27.4 \mathrm{kDa})$. In previous studies, the Mw of chitosanases from GH46 and GH75 families has mostly been found to range from 23.0 to $35.0 \mathrm{kDa}$, whereas chitosanases from GH3 and GH8 families usually exhibited an $\mathrm{Mw}$ greater than $40.0 \mathrm{kDa}$ (Table 1). Furthermore, the $\mathrm{Mw}$ of Csn-SH was similar to those of chitosanases from Bacillus.

\section{Characteristics of Csn-SH}

Chitosan precipitates in solution when the $\mathrm{pH}$ was greater than 6.0 , so buffer with a $\mathrm{pH}$ lower than 6.0 was selected for the optimal $\mathrm{pH}$ study. The optimal $\mathrm{pH}$ levels for chitosanases were reported in previous studies mainly between 4.0 and 8.0 (Table 1), and the optimal pH of Csn-SH was 5.0 (Figure 2A). The activity of Csn-SH remained greater than $86.70 \%$ of its maximum value at $\mathrm{pH} 5.5$ and $89.35 \%$ at $\mathrm{pH} 7.0$ to 8.0 under $1 \mathrm{~h}$ of incubation at $45^{\circ} \mathrm{C}$ (Figure 2B). Additionally, Csn-SH showed high activity at temperatures ranging from 20 to $55^{\circ} \mathrm{C}$ and exhibited the maximum activity at $45^{\circ} \mathrm{C}$. Notably, 78.11 and $83.57 \%$ of the maximum activity were observed at 20 and $25^{\circ} \mathrm{C}$, respectively (Figure 2C). After incubation for 0.5 to $2 \mathrm{~h}$, Csn-SH retained more than 92.84 and $91.43 \%$ of its original activity at 20 and $30^{\circ} \mathrm{C}$, respectively. However, it was rapidly inactivated at $40^{\circ} \mathrm{C}$, retaining $58.92 \%$ of its activity when incubated at $40^{\circ} \mathrm{C}$ for $1 \mathrm{~h}$ (Figure 2D). In particular, Csn-SH was stable after incubation at $30^{\circ} \mathrm{C}$ for $2 \mathrm{~h}$, whereas CsnB from Bacillus sp. BY01 lost 50\% of its activity when incubated at $30^{\circ} \mathrm{C}$ for $20 \mathrm{~min}$ (Yang et al., 2019), and CsnS from Serratia sp. QD07 retained $80.0 \%$ of its initial activity at $30^{\circ} \mathrm{C}$ for $2 \mathrm{~h}$ (Zheng et al., 2021).

In order to maximize enzyme activity and utilization efficiency, chitosanase is stable in high temperature, and alkaline conditions are often selected in industry. However, few chitosanases could meet the requirements. Wang et al. (2018) immobilized chitosanase on the surface of $\mathrm{Fe}_{3} \mathrm{O}_{4}-\mathrm{SiO}_{2}$ magnetite nanoparticles through covalent binding, which not only significantly increased the catalytic efficiency, but also improved the thermostability $11.0 \%$ and recycling times of chitosanase (Wang et al., 2018). Similar results were reported for amylase (Atiroglu et al., 2021). In addition, site-directed mutation (Yun et al., 2006) and fusion with related protein modules (Han et al., 2017) have been shown to improve the thermal stability of chitosanase as well. In this study, Csn-SH showed an optimal temperature at $45^{\circ} \mathrm{C}$, and it was stable at $\mathrm{pH} 7.0$ to 8.0. Thus, Csn$\mathrm{SH}$ is a promising candidate for COSs preparation in industrial scale by improving its thermostability in further study.

The effects of different metal ions and chemical ( 1 and $5 \mathrm{mM}$ final concentrations) on the activity of Csn-SH were also determined (Table 2); 1 and $5 \mathrm{mM} \mathrm{Mn}^{2}+$ dramatically enhanced the activity of Csn-SH by 47.1 to $64.6 \%$, respectively; $5 \mathrm{mM} \mathrm{Mn}^{2}+$ enhanced the activity of CsnB by 2.6 -fold with respect to its initial activity (Yang et al., 2019), and $10 \mathrm{mM}$ $\mathrm{Mn}^{2}+$ increased the activity of Csn21c 2-fold (Guo et al., 2019). The activities of chitosanases could be enhanced by $\mathrm{Mn}^{2}+$ at different concentrations. The reason might be related to the different strain sources of chitosanases and the metal ion-binding sites in the chitosanase structure (Nguyen et al., 2014; Yang et al., 2019). However, most of the metal ions inhibited the activity of Csn-SH. In this study, the inhibitory effects of $\mathrm{Cu}^{2}+, \mathrm{Zn}^{2}+$, $\mathrm{Fe}^{3+}$, and $\mathrm{Al}^{3+}$ on Csn-SH activity were significantly greater than those of $\mathrm{Na}^{+}, \mathrm{Li}^{+}, \mathrm{Mg}^{2}+, \mathrm{Fe}^{2+}, \mathrm{Co}^{2+}$, and $\mathrm{Pb}^{2+}$. $\mathrm{Cu}^{2}+$ caused the strongest inhibition of Csn-SH enzymatic 

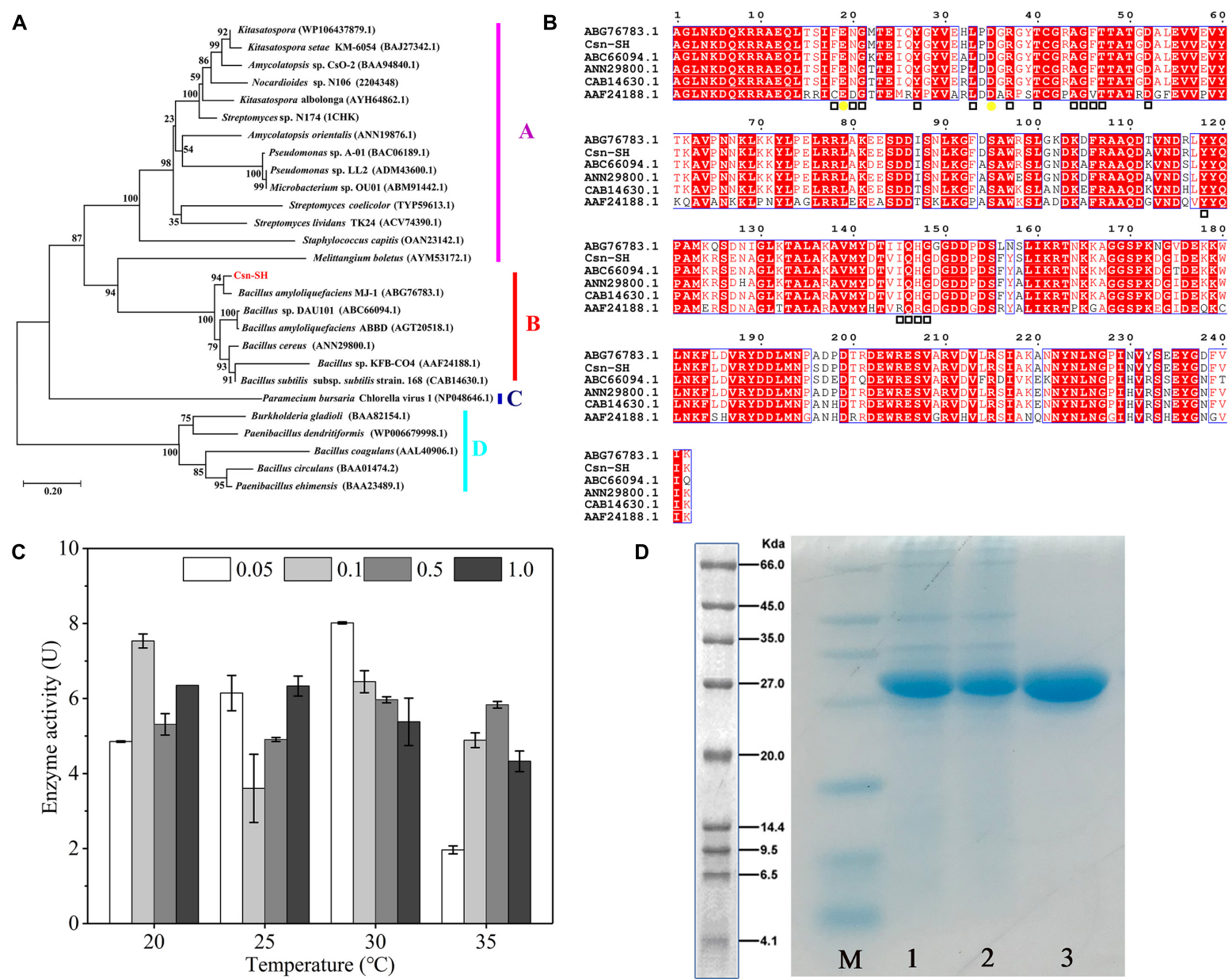

FIGURE 1 | Sequence analysis, expression, and purification of Csn-SH. (A) The neighbor-joining tree shows phylogenetic relationships between Csn-SH and other GH46 family members. The scale bar indicates the average number of amino acid substitutions per site. The bootstrap test of the tree was performed with 1,000 replications. (B) Sequence alignment of Csn-SH with other GH46 family members. The catalytic sites and sugar-binding sites were marked with yellow circles and hollow squares, respectively. The sequence of Csn-SH did not include the 32-amino-acid residues (signal peptide) at N-terminus. (C) The enzyme activities of Csn-SH induced by different concentrations of IPTG at different temperatures, whereas $0.05,0.1,0.5$, and 1.0 indicated the concentration (mM) of IPTG.

(D) SDS-PAGE analysis of purified Csn-SH. M, molecular marker; 1 and 2, crude enzymes induced by $0.05 \mathrm{mM} \mathrm{IPTG}$ at $30^{\circ} \mathrm{C}$; 3, purified Csn-SH.

TABLE 1 | The characteristics of chitosanases from different sources.

\begin{tabular}{lllccccc}
\hline Name & Sources & Family & Mw (kDa) & pH & Temperature ('C) & Major products & References \\
\hline Chitosanase & Anabaena fertilissima & GH3 & 41.0 & - & - & DP2-3 & Gupta et al., 2010 \\
Chitosanase & Bacillus sp. TS & GH8 & 47.0 & 5.0 & 60 & DP3-6 & Zhou et al., 2015 \\
Csn-SH & Bacillus atrophaeus BSS & GH46 & 27.0 & 5.0 & 45 & DP2-4 & This study \\
Csn & Bacillus subtilis 168 & GH46 & 30.0 & 5.5 & 50 & DP2-4 & Pechsrichuang et al., 2013 \\
BaCsn46A & Bacillus amyloliquefaciens & GH46 & 29.7 & 6.0 & 50 & DP2-10 & Qin et al., 2018 \\
Csn-CAP & Staphylococcus capitis & GH46 & 35.0 & 7.0 & 30 & DP2-3 & Sun et al., 2018 \\
CsnB & Bacillus sp. BY01 & GH46 & 30.0 & 5.0 & 35 & DP2-3 & Yang et al., 2019 \\
Csn-BAC & Bacillus sp. MD-5 & GH46 & 35.0 & 7.0 & 40 & DP2-3 & Yang et al., 2020 \\
CsnQ & Bacillus sp. Q1098 & GH46 & 30.0 & 5.3 & 60 & DP2 & Ma et al., 2020 \\
BaCsn46B & Bacillus amyloliquefaciens ECU08 & GH46 & 29.0 & 6.5 & 55 & DP2-3 & Luo et al., 2020 \\
CSN4 & Marine mud metagenomic DNA & GH46 & 26.0 & 7.0 & 30 & DP2-4 & Sun et al., 2020 \\
BbCSN-1 & Beauveria bassiana & GH75 & 33.0 & 5.0 & 30 & DP2-3 & Liu et al., 2020 \\
Csn75 & Aspergillus fumigatus CJ22-326 & GH75 & 23.5 & 5.0 & 55 & DP2-4 & Zhou et al., 2020
\end{tabular}



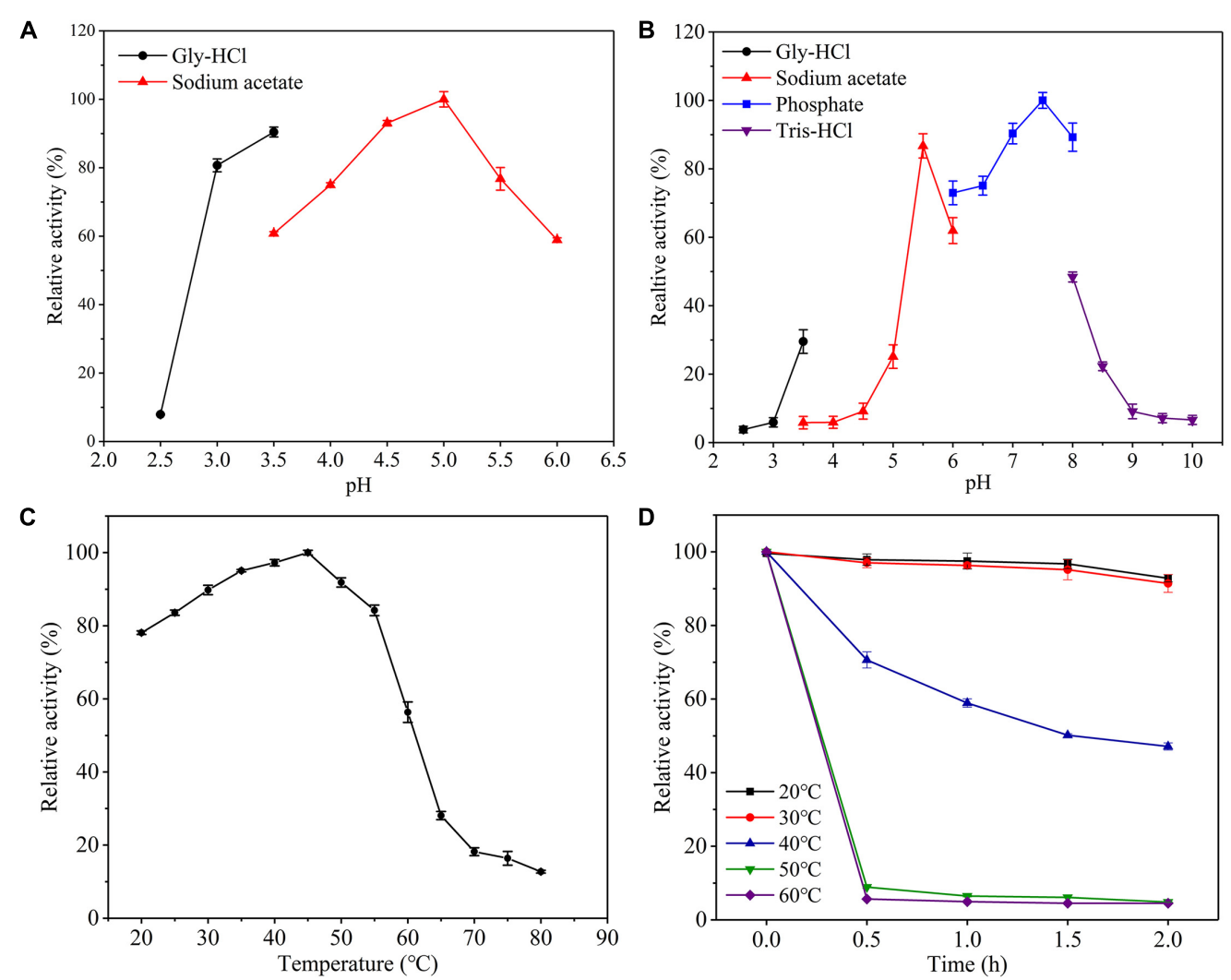

FIGURE 2 | Effect of $\mathrm{pH}$ and temperature on the activity and stability of the purified Csn-SH. (A,B) Optimal pH and pH stability. (C,D) Optimal temperature and thermostability. The initial catalytic activity without preincubation was used as control, which was defined as $100 \%$.

TABLE 2 | Effects of different chemicals on the enzyme activity of purified Csn-SH.

\begin{tabular}{lll}
\hline Chemicals & \multicolumn{2}{c}{ Relative activity (\%) } \\
\cline { 2 - 3 } & $\mathbf{1} \mathbf{~ m M}$ & $\mathbf{5 ~} \mathbf{~ M}$ \\
\hline Control & $100 \pm 3.07$ & $100 \pm 1.04$ \\
$\mathrm{Na}^{+}$ & $80.38 \pm 0.50$ & $86.51 \pm 1.80$ \\
$\mathrm{~K}^{+}$ & $90.41 \pm 1.50$ & $101.25 \pm 3.53$ \\
$\mathrm{Li}^{+}$ & $79.57 \pm 2.44$ & $88.17 \pm 3.53$ \\
$\mathrm{Mg}^{2+}$ & $93.73 \pm 1.82$ & $95.85 \pm 3.81$ \\
$\mathrm{Ca}^{2+}$ & $96.12 \pm 2.32$ & $97.65 \pm 2.01$ \\
$\mathrm{Cu}^{2+}$ & $0 \pm 0$ & $0 \pm 0$ \\
$\mathrm{Mn}^{2+}$ & $147.14 \pm 3.70$ & $164.55 \pm 3.46$ \\
$\mathrm{Zn}^{2+}$ & $41.08 \pm 1.82$ & $11.37 \pm 0.42$ \\
$\mathrm{Fe}^{2+}$ & $82.76 \pm 1.00$ & $72.26 \pm 0.55$ \\
$\mathrm{Co}^{2+}$ & $81.57 \pm 0.56$ & $92.05 \pm 0.28$ \\
$\mathrm{~Pb}^{2+}$ & $85.90 \pm 0.01$ & $88.24 \pm 4.36$ \\
$\mathrm{Fe}^{3+}$ & $23.53 \pm 1.44$ & $7.08 \pm 2.84$ \\
$\mathrm{Al}^{3+}$ & $57.32 \pm 3.38$ & $9.71 \pm 2.84$ \\
$\mathrm{EDTA}^{2+}$ & $84.90 \pm 2.13$ & $74.96 \pm 4.15$ \\
\hline
\end{tabular}

activity (Table 2). The differences in the inhibitory effects of different metal ions on Csn-SH activity might be associated with the presence of a larger number of amino groups on the chitosan surface, which complexed with transition metal ions and thus decreased the enzyme activity (Brunel et al., 2013). In addition, 1 and $5 \mathrm{mM}$ EDTA inhibited the activity of Csn-SH by 15.10 and $25.04 \%$, respectively (Table 2), possibly because the $-\mathrm{COOH}$ group of EDTA and $-\mathrm{NH}_{2}$ on the chitosan surface can form an amide bond in a concentration-dependent manner in acidic solution (Gyliene et al., 2006). In accordance with our results, $5 \mathrm{mM}$ EDTA has been shown to dramatically inhibit the activities of CsnB (Bacillus sp. BY01) (Yang et al., 2019) and CsnQ (Bacillus sp. Q1098) (Ma et al., 2020).

\section{Substrate Specificity and Kinetic Parameters of Csn-SH}

Colloidal chitin, CMC, colloidal chitosan (DD $\geq 85 \%$ ), and water-soluble chitosan (DD $\geq 95 \%$ ) were used as substrates to investigate the substrate specificity of Csn-SH. The results demonstrated that the hydrolytic activity of Csn$\mathrm{SH}$ toward chitosan was directly proportional to the DD (Supplementary Table 1). Nevertheless, Csn-SH exhibited no activity toward chitin or CMC (Supplementary Table 1), which was in accordance with the reported findings for other chitosanases, such as Csn-BAC from Bacillus sp. MD-5 (Yang et al., 2020) and Csn-CAP from Staphylococcus capitis (Sun et al., 2018).

The kinetic parameters of Csn-SH were determined under the optimal conditions. The results presented in 
Supplementary Figure 1 showed that the $K_{m}$ and $V_{\max }$ values of Csn-SH were $0.50 \mathrm{mg} / \mathrm{mL}$ and $140.05 \mu \mathrm{mol}$ $\mathrm{mg}^{-1} \mathrm{~min}^{-1}$, respectively. The $K_{m}$ and $V_{\text {max }}$ of Csn21c from Streptomyces albolongus were $7.4 \mathrm{mg} / \mathrm{mL}$ and 263.1 $\mu \mathrm{mol} \mathrm{mg} \mathrm{min}^{-1}$, respectively (Guo et al., 2019), and the corresponding values of Csn from B. subtilis were $1.57 \mathrm{mg} / \mathrm{mL}$ and $8.83 \mu \mathrm{mol} \mathrm{mg}^{-1} \mathrm{~min}^{-1}$, respectively (Pechsrichuang et al., 2013). The $K_{m}$ value of Csn-SH was much lower than those of the above chitosanases, and the $V_{\max }$ was higher than those of these chitosanases, which indicated that Csn$\mathrm{SH}$ presented high substrate affinity and catalytic efficiency (Zhou et al., 2020).

\section{Hydrolysis Pattern of Csn-SH}

The hydrolysis properties of Csn-SH toward colloidal chitosan (1-4\%, wt/vol) were analyzed by TLC and ESI-MS (Figure 3). In $20 \mathrm{~min}$, Csn-SH could effectively hydrolyze $1-4 \%$ chitosan to yield COSs [mainly of $(\mathrm{GlcN})_{2-6}$ ]. With extension of the reaction time, DP5 and DP6 were further degraded into DP2-4, whereas DP4 was not hydrolyzed further (Figures 3A-D). The hydrolysates of chitosan after $24 \mathrm{~h}$ of incubation at $45^{\circ} \mathrm{C}$ were also detected. ESI-MS analysis showed that $1-4 \%$ of the chitosan hydrolysates mainly consisted of $(\mathrm{GlcN})_{2-4}$, with a small amount of $(\mathrm{GlcN})_{5-6}$ (Figures 3E-H), which was consistent with the TLC results.

To shed light on the cleavage pattern of Csn-SH, $(\mathrm{GlcN})_{2-6}$ were degraded by $\mathrm{Csn}-\mathrm{SH}$, and the hydrolysates were also analyzed by TLC. The results showed that Csn-SH could not hydrolyze $(\mathrm{GlcN})_{2-4}$ (Supplementary Figures 2A-C), whereas $(\mathrm{GlcN})_{5}$ could be degraded into $(\mathrm{GlcN})_{2-3}$ (Supplementary Figure 2D). For $(\mathrm{GlcN})_{6}$ substrates, $(\mathrm{GlcN})_{2-4}$ products were obtained (Supplementary Figure 2E). CSN4 from a marine mud metagenome could degrade $(\mathrm{GlcN})_{6}$ into $(\mathrm{GlcN})_{2}$ and $(\mathrm{GlcN})_{4}$ without $(\mathrm{GlcN})_{3}$, which demonstrated that CSN4 preferred to digest substrates asymmetrically rather than symmetrically (Sun et al., 2020). Therefore, the above results indicated that Csn-SH exhibits no preference for substrate symmetry. Additionally, the hydrolysis properties of Csn-SH were similar to those of other members of GH46 family chitosanases, such as Csn from B. subtilis 168 (Pechsrichuang et al., 2013) and Csn-CAP from S. capitis (Sun et al., 2018), which yielded COSs with DP2-3 or DP2-4 (Table 1). These results revealed that $\mathrm{Csn}-\mathrm{SH}$ could hydrolyze chitosan
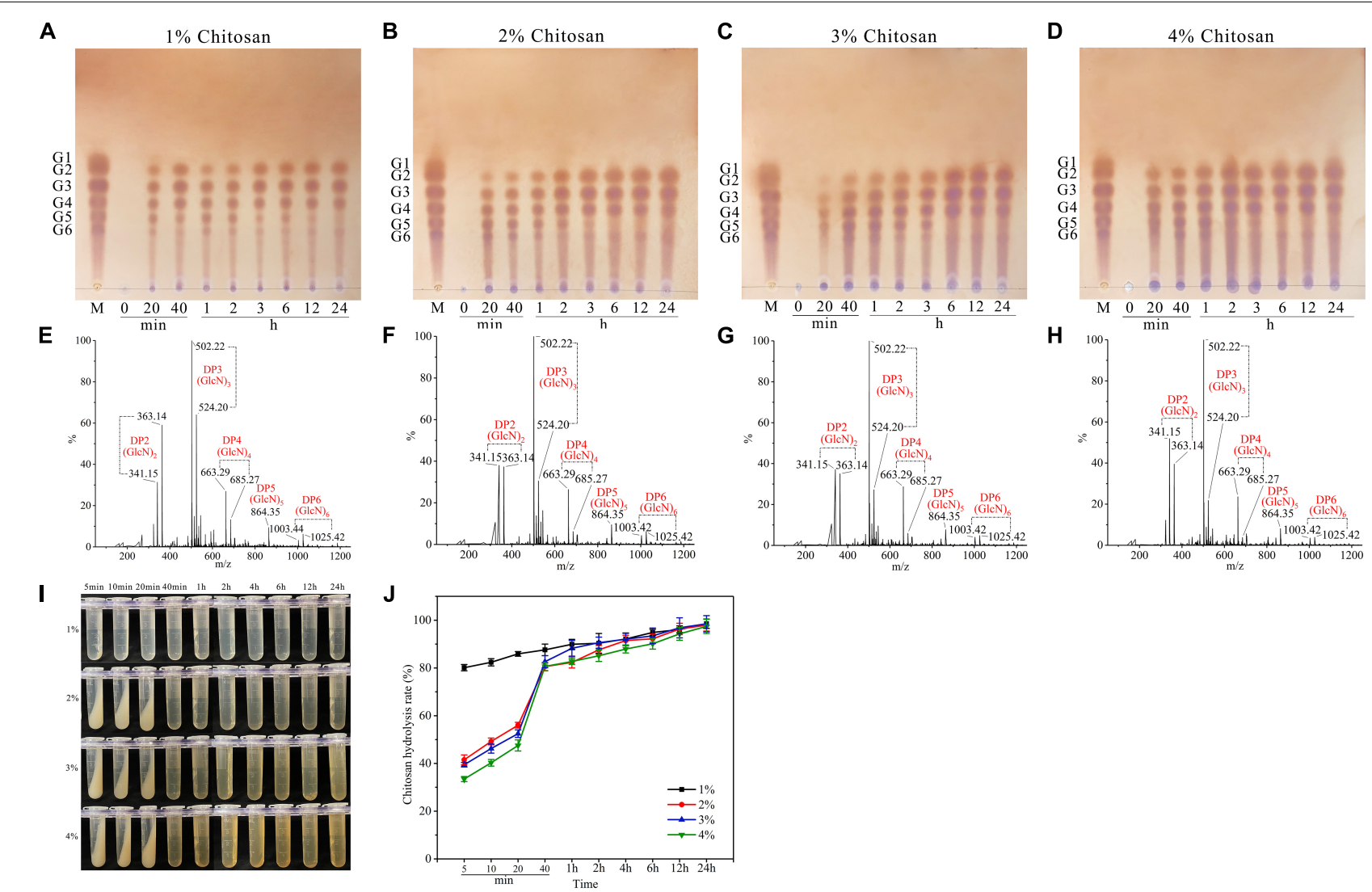

FIGURE 3 | TLC, ESI-MS analysis, and yield of chitosan hydrolysates prepared with Csn-SH. (A-D) TLC and ESI-MS analysis of 1-4\% (wt/vol) chitosan hydrolysates for different reaction time, respectively. G1-G6 indicated $(\mathrm{GlcN})_{1}$ to $(\mathrm{GlcN})_{6}$. Lane M: standard chitooligomers. Lanes 0-24: Chitosan hydrolysates prepared with Csn-SH incubated at $45^{\circ} \mathrm{C}$ for 20 min, 40 min, 1, 2, 3, 6, 12, and 24 h, respectively. (E-H) ESI-MS analysis of 1-4\% chitosan hydrolysates prepared with Csn-SH at $45^{\circ} \mathrm{C}$ for $24 \mathrm{~h}$, respectively. (I) The precipitation of undegraded chitosan with $20 \mathrm{U} / \mathrm{mL}$ crude Csn-SH at different reaction time. (J) The hydrolysis rates of $1-4 \%$ chitosan by Csn-SH at different reaction time with $20 \mathrm{U} / \mathrm{mL}$ crude Csn-SH. 
in an endo-type pattern and recognize $(\mathrm{GlcN})_{5}$ as the minimal substrate.

\section{Economic and Efficiency Analysis of Csn-SH}

It is worth noting that the hydrolysis rate of $1-4 \%$ chitosan by Csn-SH $(20 \mathrm{U} / \mathrm{mL}$, final concentration) reached $80.57 \%$ within $40 \mathrm{~min}$ (Figure 3J). To evaluate whether Csn-SH is cleaner and more efficient than chitosanases and commercial enzymes reported previously, we compared the characteristics of chitosanases, commercial cellulase, lipase, papain, pectinase, and pepsin during the hydrolytic processes of chitosan; the examined characteristics included the dosage of enzymes, water consumption, and the value of hydrolysates and resulting profits (Table 3). The characteristics of the above enzymes were summarized according to previous publications (Lin et al., 2002; Cabrera and Van Cutsem, 2005; Roncal et al., 2007; Lee et al., 2008; Tegl et al., 2016; Zhou et al., 2020). One ton of colloidal chitosan was used as the raw material, and the corresponding concentration was obtained by using $1 \%$ acetic acid. The profits were calculated based on the raw chitosan materials and the yield of COSs, except for the costs of water and enzymes. The conversion of chitosan and COS prices was calculated based on the data (Deng et al., 2020). The results presented in Table 3 showed that Csn-SH produced the highest profits during the degradation of chitosan, followed by lipase, pepsin, Csn75, and cellulase. The output values of the COSs prepared with pectinase and papain were lower than the input values of the chitosan material. It was

TABLE 3 | The properties and profits of different enzymes for the conversion of chitosan to chitooligosaccharides (COSs).

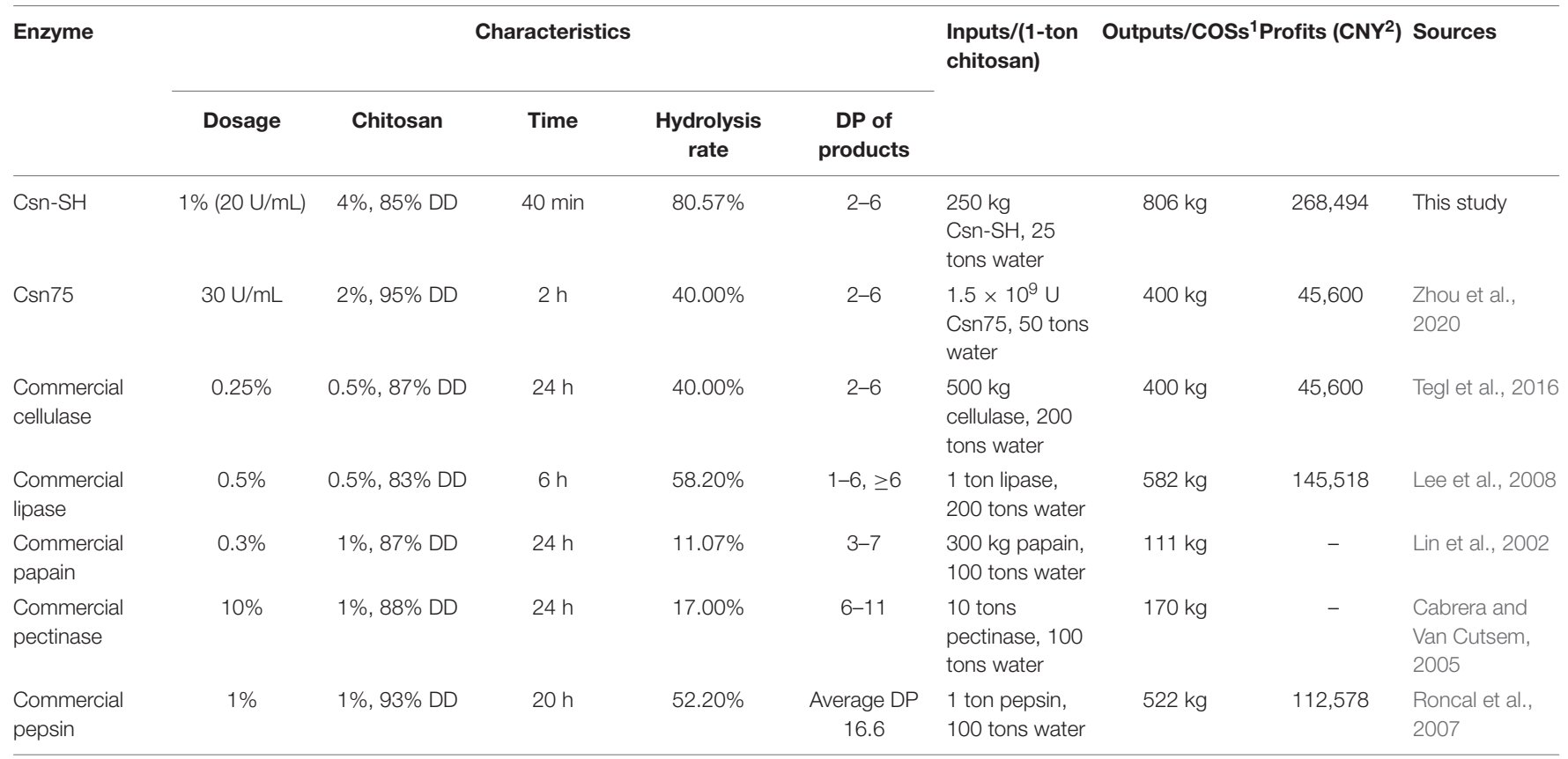

${ }^{1}$ The price of chitosan and COSs were 174 and $550 \mathrm{CNY} / \mathrm{kg}$, respectively. ${ }^{2} \mathrm{CNY}$ indicates China Yuan. The profit was the COSs' values minus the chitosan costs.
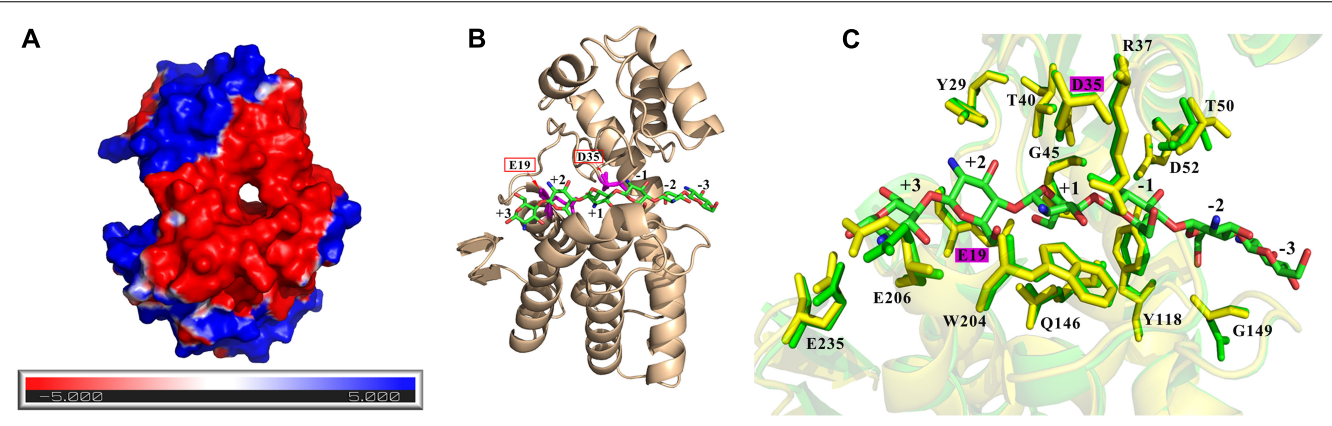

FIGURE 4 | Homology modeling structure and docking of Csn-SH. (A) The electrostatic-potential surface of Csn-SH. (B) The overall structural view of Csn-SH in complex with $(\mathrm{GlcN})_{6}$. (GlcN)6 was labeled -4 to +2 from the nonreducing end to the reducing end. Two catalytic residues (E19 and D35) of Csn-SH were colored by magenta. (C) Substrate interaction of $\mathrm{Csn}-\mathrm{SH}$. The residue numbers of Csn-SH represented the sequence without 32 amino acids (signal peptide) at $\mathrm{N}$-terminal. The amino residues from Csn-SH and chitosanase CsnMY002 (PDB: 7C6C) are colored by yellow and green, respectively. 
noteworthy that among these enzymes, Csn-SH took only $40 \mathrm{~min}$ to complete the same process, for which lipase and pepsin required 6 and $20 \mathrm{~h}$, respectively. In addition, the amount of water consumption by Csn-SH was also the lowest, at only $25 \%$ and $50 \%$ of the amounts required by lipase and pepsin, respectively (Table 3 ). The above results indicated that Csn$\mathrm{SH}$ could reduce the waste of water resources and decrease hydrolysis time during the degradation of chitosan relative to other enzymes.

\section{Homology Modeling and Molecular Docking}

A three-dimensional model of Csn-SH was generated by homology modeling using the crystal structure of $B$. subtilis MY002 (PDB: 7C6C; identity: 91.3\%) as a template (Li et al., 2021). Chitosan is a natural cationic polysaccharide, so the substrate-binding sites of chitosanase are highly negatively charged (Takasuka et al., 2014). As shown in Figure 4A, the substrate-binding region of $\mathrm{Csn}-\mathrm{SH}$ formed a highly negatively charged closed tunnel (Figure 4A), which differed to the open clefts structure of other GH46 chitosanases (Marcotte et al., 1996; Li et al., 2021). Tunnel-like substrate-binding sites lined with aromatic residues often exhibited in the structure of processive enzymes, which could provide a hydrophobic sheath to facilitate substrate sliding in the tunnel (Varrot et al., 2003; Li et al., 2021). Conserve domain database analysis revealed that $\mathrm{Csn}-\mathrm{SH}$ was a monomeric enzyme belonging to the lysozyme superfamily. It was composed of two lobes connected by a long bent $\alpha$-helix and a hinge segment (Figure 4B). The results were similar to those obtained for CsnMY002 from B. subtilis (Li et al., 2021), SACTE_5457 from Streptomyces (Takasuka et al., 2014), and BaCsn46B from B. amyloliquefaciens (Luo et al., 2020).

Molecular docking analysis was performed to further explore the interaction of Csn-SH and $(\mathrm{GlcN})_{6}$. The substrate was located in the closed tunnel of Csn-SH, and $(\mathrm{GlcN})_{6}$ was labeled -4 to +2 from the nonreducing end to the reducing end (Figure 4B). Two highly conserved catalytic residues, E19 and D35, were identified as the general acid/base and nucleophilic catalytic residues of Csn-SH, respectively, which were located on either side of the substrate (Figure 4B). During the reaction, E19 protonated glycosidic oxygen, and D35 polarized the attacking water to complete hydrolysis by using an "inverting" catalytic mechanism (Marcotte et al., 1996). The presumptive cleavage site of Csn-SH was matched to the glycosidic bond between the -1 to +1 subsite (Figure 4B), which suggested that Csn-SH might cleave $(\mathrm{GlcN})_{6}$ into $(\mathrm{GlcN})_{2}$ and $(\mathrm{GlcN})_{4}$. This speculation was confirmed by the result of TLC, which showed that the hydrolysates prepared with Csn-SH contained $(\mathrm{GlcN})_{2}$ and $(\mathrm{GlcN})_{4}$. It was noteworthy that $(\mathrm{GlcN})_{3}$ was also found in the hydrolysates and considered as the dominant product (Figure 3 and Supplementary Figure 2E). The results implied that another putative cleavage site might locate between the -2 and -1 position (Figure $4 \mathrm{C}$ ); thus, Csn-SH could cleave $(\mathrm{GlcN})_{6}$ into two $(\mathrm{GlcN})_{3}$ molecules. Furthermore, the docking and TLC results of Csn-SH were consistent with the results of chitosanase OU01 (Lyu et al., 2014) and CsnMY002 (Li et al., 2021). However, the hydrolysates from CsnMY002 mainly contained $(\mathrm{GlcN})_{2}$ and $(\mathrm{GlcN})_{3}$ without $(\mathrm{GlcN})_{4}$, which might be related to the difference of enzyme dosage in the reaction mixture.

In addition, results from Figure 4C showed that the amino acid residues and catalytic residues in Csn-SH superimposed well with the corresponding residues in the CsnMY002 (PDB: 7C6C) structure. The sugar units and adjacent residues (Y29, R37, G45, T50, D52, Y118, Q146, G149, W204, E206, and E235) could form a hydrogen-bond network to stabilize the substrate (Li et al., 2021). Moreover, the hydrogen bonding between Y29, Y118, W204, and the sugar units might inhibit the substrate sliding in the substrate-binding tunnel (Zakariassen et al., 2009). Nevertheless, the functions of these corresponding key residues in Csn-SH remained to be further investigated. The above results proposed that Csn-SH might cleave glycosidic bonds of chitosan between subsites -2 and +1 as a nonprocessive enzyme (Lyu et al., 2015; Li et al., 2021). However, more studies need to explore the action mode.
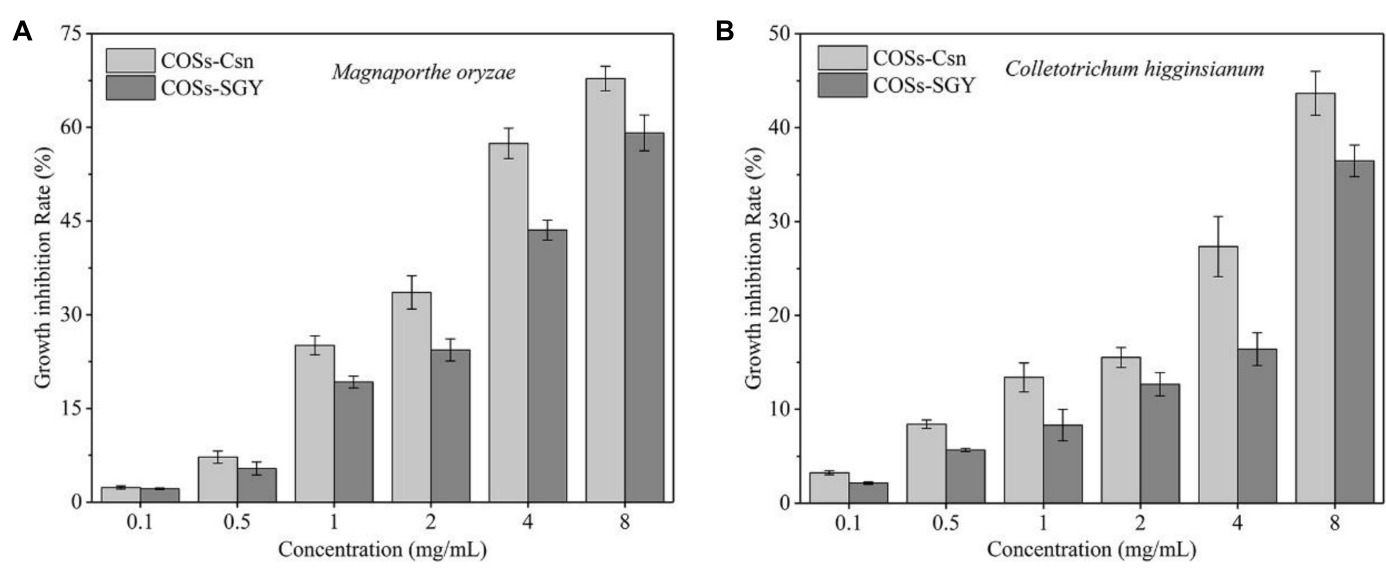

FIGURE 5 | The fungal mycelia generation inhibition rate affected by COSs at different concentrations. (A) Inhibition of mycelia generation in M. oryzae treated with COSs at different concentrations. (B) Inhibition of mycelia generation in C. higginsianum treated with COSs at different concentrations. COSs-Csn indicated the COSs prepared with Csn-SH, whereas COSs-SGY represented the COSs purchased from Guangzhou SGY Agricultural Science and Technology Co., Ltd. 


\section{Antifungal Activity of COSs}

The inhibition of COSs on phytopathogenic fungi M. oryzae and C. higginsianum are shown in Figure 5. M. oryzae and C. higginsianum have been reported to cause severe damage to rice (Griffith et al., 2021) and various cruciferous plants, respectively (Yan et al., 2020). COS treatments significantly inhibited the growth of the M. oryzae and C. higginsianum, but had a stronger inhibitory effect on $M$. oryzae. The inhibition effects became more significant as the concentration increases, which displayed a dose-dependent inhibition (Figure 5). The $\mathrm{EC}_{50}$ of $M$. oryzae and C. higginsianum with COSs-Csn treatment were 4.84 and $8.47 \mathrm{mg} / \mathrm{mL}$, respectively, while the values with COSs-SGY treatment were 6.06 and $9.91 \mathrm{mg} / \mathrm{mL}$, respectively. These results were consistent with previous study showing that COSs exhibited good antifungal activity (Wang et al., 2021b), because the cationic $-\mathrm{NH}_{2}$ groups of COSs can form ammonium groups by absorbing $\mathrm{H}^{+}$, and then bind to negatively charged components of the microbial cell wall, resulting in agminated microbes and further lysis (Liaqat and Eltem, 2018).

However, antifungal activity of COSs is significantly affected by the DP, degree of acetylated and microorganism types (Liaqat and Eltem, 2018). Among them, DP $\geq 5$ was essential for antimicrobial activity of fully deacetylated COS (Li et al., 2014). The growth inhibition rate (27.35\%) of M. oryzae with $4 \mathrm{mg} / \mathrm{mL}$ COSs-Csn treatment (Figure 5A) was higher than CoA-COSs (20.0\%) in a previous study (Wang et al., 2021b); the reason may be related to the difference of DP. COSs-Csn was composed of DP2-6, whereas CoA-COSs consisted of DP3-5. However, the antifungal activity of COSs-SGY (DP2-7) was lower than COSs-Csn (DP2-6), which might be due to the relative content of oligosaccharides with different DP (Sanchez et al., 2017). Previous research pointed out that COSs (DP2-6) could restore the activities of endogenous antioxidants and inhibit intracellular ROS, which played great potential for the oxidative disease treatment (Liu et al., 2009). Therefore, COSs prepared with CsnSH have broad application prospects in the fields of antifungal and antioxidative agents, and other active functions need to be further studied.

\section{CONCLUSION}

Our results suggested that Csn-SH is a biotechnologically potential chitosanase characterized from B. atrophaeus BSS. Csn-SH showed optimal activity under the condition of $45^{\circ} \mathrm{C}$ and $\mathrm{pH} 5.0$, and it was stable at $\mathrm{pH} 7.0-8.0$, below $30^{\circ} \mathrm{C}$.

\section{REFERENCES}

Atiroglu, V., Atiroglu, A., and Ozacar, M. (2021). Immobilization of alpha-amylase enzyme on a protein @metal-organic framework nanocomposite: a new strategy to develop the reusability and stability of the enzyme. Food Chem. 349:129127. doi: 10.1016/j.foodchem.2021.129127

Benchamas, G., Huang, G. L., Huang, S. Y., and Huang, H. L. (2021). Preparation and biological activities of chitosan oligosaccharides. Trends Food Sci. Tech. 107, 38-44. doi: 10.1016/j.tifs.2020.11.027
$(\mathrm{GlcN})_{5}$ was the minimally recognized substrate of Csn-SH. Under the optimal reaction condition, Csn-SH hydrolyzed colloidal chitosan (concentration $\geq 1 \%$ ) into DP2-6 in an endotype pattern. Importantly, Csn-SH could reduce the waste of water resources and save hydrolysis time during the hydrolytic processes of chitosan. The hydrolysates prepared with Csn$\mathrm{SH}$ could inhibit the growth of fungi. The excellent hydrolytic capabilities make Csn-SH a perfect candidate for the efficient production of COSs that could be used as biocontrol agents.

\section{DATA AVAILABILITY STATEMENT}

The datasets presented in this study can be found in online repositories. The names of the repository/repositories and accession number(s) can be found below: https://www.ncbi.nlm. nih.gov/genbank/, CP007640.

\section{AUTHOR CONTRIBUTIONS}

DC: investigation, data curation, analysis, writing-original draft preparation, and conceptualization. JY: investigation, review and editing, and conceptualization. BL: investigation. HS: conceptualization, project administration, review and editing, and funding acquisition. All authors have read and agreed to the published version of the manuscript.

\section{FUNDING}

This work was supported by the National Key Research and Development Program of China (2016YFD0200405-5) and the Science and Technology Planning Project of Guangdong (2019B030301007).

\section{SUPPLEMENTARY MATERIAL}

The Supplementary Material for this article can be found online at: https://www.frontiersin.org/articles/10.3389/fmicb. 2021.682829/full\#supplementary-material

Supplementary Figure 1 | The Lineweaver-Burk plot of the Csn-SH activity.

Supplementary Figure 2 | TLC analysis of $(\mathrm{GlCN})_{2-6}$ hydrolysates prepared with Csn-SH.

Supplementary Table 1 | Substrate specificity of purified Csn-SH.

Brunel, F., El Gueddari, N. E., and Moerschbacher, B. M. (2013). Complexation of copper(II) with chitosan nanogels: toward control of microbial growth. Carbohyd. Polym. 92, 1348-1356. doi: 10.1016/j.carbpol.2012.10.025

Cabrera, J. C., and Van Cutsem, P. (2005). Preparation of chitooligosaccharides with degree of polymerization higher than 6 by acid or enzymatic degradation of chitosan. Biochem. Eng. J. 25, 165-172. doi: 10.1016/j.bej.2005.04.025

Deng, J. J., Mao, H. H., Fang, W., Li, Z. Q., Shi, D., Li, Z. W., et al. (2020). Enzymatic conversion and recovery of protein, chitin, and astaxanthin from shrimp shell waste. J. Clean. Prod. 271:122655. doi: 10.1016/j.jclepro.2020.122655 
Dutta, J., Tripathi, S., and Dutta, P. K. (2012). Progress in antimicrobial activities of chitin, chitosan and its oligosaccharides: a systematic study needs for food applications. Food Sci. Techno. Int. 18, 3-34. doi: 10.1177/1082013211399195

Griffith, A., Wise, P., Gill, R., Paukett, M., Donofrio, N., and Seyfferth, A. L. (2021). Combined effects of arsenic and Magnaporthe oryzae on rice and alleviation by silicon. Sci. Total Environ. 750:142209. doi: 10.1016/j.scitotenv.2020.142209

Guo, N., Sun, J. N., Wang, W., Gao, L., Liu, J. B., Liu, Z., et al. (2019). Cloning, expression and characterization of a novel chitosanase from Streptomyces albolongus ATCC 27414. Food Chem. 286, 696-702. doi: 10.1016/j.foodchem. 2019.02.056

Gupta, V., Prasanna, R., Natarajan, C., Srivastava, A. K., and Sharma, J. (2010). Identification, characterization, and regulation of a novel antifungal chitosanase gene (cho) in Anabaena spp. Appl. Environ. Microb. 76, 2769-2777. doi: 10. 1128/Aem.02673-09

Gyliene, O., Nivinskiene, O., and Razmute, I. (2006). Copper(II)-EDTA sorption onto chitosan and its regeneration applying electrolysis. J. Hazard. Mater. 137, 1430-1437. doi: 10.1016/j.jhazmat.2006.04.044

Han, Y., Gao, P., Yu, W., and Lu, X. (2017). Thermostability enhancement of chitosanase CsnA by fusion a family 5 carbohydrate-binding module. Biotechnol. Lett. 39, 1895-1901. doi: 10.1007/s10529-017-2406-2

Hong, S. K., and Kang, D. K. (2006). Molecular cloning and characterization of chitosanase gene from Bacillus amyloliquefaciens MJ-1. Korean J. Microbiol. 42, $142-148$.

Jiang, Z. W., Wang, S. N., Hou, J., Chi, J. H., Wang, S., Shao, K., et al. (2020). Effects of carboxymethyl chitosan oligosaccharide on regulating immunologic function and inhibiting tumor growth. Carbohyd. Polym. 250:116994. doi: 10. 1016/j.carbpol.2020.116994

Lee, D. X., Xia, W. S., and Zhang, J. L. (2008). Enzymatic preparation of chitooligosaccharides by commercial lipase. Food Chem. 111, 291-295. doi: 10.1016/j.foodchem.2008.03.054

Li, Y., Gou, Y., Liu, Z. C., Xie, T., and Wang, G. G. (2021). Structure-based rational design of chitosanase CsnMY002 for high yields of chitobiose. Colloid. Surface B 202:111692. doi: 10.1016/j.colsurfb.2021.111692

Li, Y., Liu, H., Xu, Q. S., Du, Y. G., and Xu, J. (2014). Chitosan oligosaccharides block LPS-induced O-GlcNAcylation of NF-kappaB and endothelial inflammatory response. Carbohydr. Polym. 99, 568-578. doi: 10. 1016/j.carbpol.2013.08.082

Liaqat, F., and Eltem, R. (2018). Chitooligosaccharides and their biological activities: a comprehensive review. Carbohydr. Polym. 184, 243-259. doi: 10. 1016/j.carbpol.2017.12.067

Lin, H., Wang, H. Y., Xue, C. H., and Ye, M. (2002). Preparation of chitosan oligomers by immobilized papain. Enzyme Microb. Tech. 31, 588-592. doi: 10.1016/S0141-0229(02)00138-2

Liu, H. T., Li, W. M., Xu, G., Li, X. Y., Bai, X. F., Wei, P., et al. (2009). Chitosan oligosaccharides attenuate hydrogen peroxide-induced stress injury in human umbilical vein endothelial cells. Pharmacol. Res. 59, 167-175. doi: 10.1016/j. phrs.2008.12.001

Liu, Y., Li, Y. L., Tong, S., Yuan, M., Wang, X. Y., Wang, J. Y., et al. (2020). Expression of a Beauveria bassiana chitosanase (BbCSN-1) in Pichia pastoris and enzymatic analysis of the recombinant protein. Protein Expres. Purif. 166:105519. doi: 10.1016/J.Pep.2019.105519

Luo, S., Qin, Z., Chen, Q. M., Fan, L. Q., Jiang, L. H., and Zhao, L. M. (2020). High level production of a Bacillus amlyoliquefaciens chitosanase in Pichia pastoris suitable for chitooligosaccharides preparation. Int. J. Bio. Macromol. 149, 1034-1041. doi: 10.1016/j.ijbiomac.2020.02.001

Lyu, Q. Q., Shi, Y. H., Wang, S., Yang, Y., Han, B. Q., Liu, W. S., et al. (2015). Structural and biochemical insights into the degradation mechanism of chitosan by chitosanase OU01. BBA-Gen. Subjects 1850, 1953-1961. doi: 10.1016/j.bbagen.2015.06.011

Lyu, Q. Q., Wang, S., Xu, W. H., Han, B. Q., Liu, W. S., Junes, D. N. M., et al. (2014). Structural insights into the substrate-binding mechanism for a novel chitosanase. Biochem. J. 461, 335-345. doi: 10.1042/Bj20140159

Ma, C. R., Li, X., Yang, K., and Li, S. Y. (2020). Characterization of a new chitosanase from a marine Bacillus sp. and the anti-oxidant activity of its hydrolysate. Mar. Drugs 18:126. doi: 10.3390/Md18020126

Marcotte, E. M., Monzingo, A. F., Ernst, S. R., Brzezinski, R., and Robertus, J. D. (1996). X-ray structure of an anti-fungal chitosanase from Streptomyces N174. Nat. Struct. Biol. 3, 155-162. doi: 10.1038/nsb0296-155
Nascimento, J. I. G., Stamford, T. C. M., Melo, N., Nunes, I. D. S., Lima, M. A. B., Pintado, M. M. E., et al. (2020). Chitosan-citric acid edible coating to control Colletotrichum gloeosporioides and maintain quality parameters of fresh-cut guava. Int. J. Biol. Macromol. 163, 1127-1135. doi: 10.1016/j.ijbiomac.2020.07. 067

Nguyen, A. D., Huang, C. C., Liang, T. W., Nguyen, V. B., Pan, P. S., and Wang, S. L. (2014). Production and purification of a fungal chitosanase and chitooligomers from Penicillium janthinellum D4 and discovery of the enzyme activators. Carbohyd. Polym. 108, 331-337. doi: 10.1016/j.carbpol.2014.02.053

Pechsrichuang, P., Yoohat, K., and Yamabhai, M. (2013). Production of recombinant Bacillus subtilis chitosanase, suitable for biosynthesis of chitosanoligosaccharides. Bioresour. Technol. 127, 407-414. doi: 10.1016/j.biortech. 2012.09.130

Qin, Z., Luo, S., Li, Y., Chen, Q. M., Qiu, Y. J., Zhao, L. M., et al. (2018). Biochemical properties of a novel chitosanase from Bacillus amyloliquefaciens and its use in membrane reactor. Lwt-Food Sci. Technol. 97, 9-16. doi: 10.1016/j.lwt.2018.06. 027

Roncal, T., Oviedo, A., Lopez de Armentia, I., Fernandez, L., and Villaran, M. C. (2007). High yield production of monomer-free chitosan oligosaccharides by pepsin catalyzed hydrolysis of a high deacetylation degree chitosan. Carbohyd. Res. 342, 2750-2756. doi: 10.1016/j.carres.2007.08.023

Sanchez, A., Mengibar, M., Rivera-Rodriguez, G., Moerchbacher, B., Acosta, N., and Heras, A. (2017). The effect of preparation processes on the physicochemical characteristics and antibacterial activity of chitooligosaccharides. Carbohyd. Polym. 157, 251-257. doi: 10.1016/j.carbpol. 2016.09.055

Silva, N. S. D., Araujo, N. K., Daniele-Silva, A., Oliveira, J. W. F., Medeiros, J. M., Araujo, R. M., et al. (2021). Antimicrobial activity of chitosan oligosaccharides with special attention to antiparasitic potential. Mar. Drugs 19:110. doi: 10.3390/ md19020110

Sun, H. H., Cao, R., Li, L. H., Zhao, L., and Liu, Q. (2018). Cloning, purification and characterization of a novel GH46 family chitosanase, Csn-CAP, from Staphylococcus capitis. Process Biochem. 75, 146-151. doi: 10.1016/j.procbio. 2018.09.021

Sun, H. H., Yang, G. S., Cao, R., Mao, X. Z., and Liu, Q. (2020). Expression and characterization of a novel glycoside hydrolase family 46 chitosanase identified from marine mud metagenome. Int. J. Biol. Macromol. 159, 904-910. doi: 10. 1016/j.ijbiomac.2020.05.147

Takasuka, T. E., Bianchetti, C. M., Tobimatsu, Y., Bergeman, L. F., Ralph, J., and Fox, B. G. (2014). Structure-guided analysis of catalytic specificity of the abundantly secreted chitosanase SACTE_5457 from Streptomyces sp. SirexAAE. Proteins 82, 1245-1257. doi: 10.1002/prot.24491

Tegl, G., Ohlknecht, C., Vielnascher, R., Kosma, P., Hofinger-Horvath, A., and Guebitz, G. M. (2016). Commercial cellulases from Trichoderma longibrachiatum enable a large-scale production of chitooligosaccharides. Pure Appl. Chem. 88, 865-872. doi: 10.1515/pac-2016-0703

Varrot, A., Frandsen, T. P., Von, I. O., Boyer, V., Cottaz, S., Driguez, H., et al. (2003). Structure basis for ligand binding and processively in cellobiohydrolase Cel6A from Humicola insolens. Strcuture 11, 855-864. doi: 10.1016/S09692126(03)00124-2

Viens, P., Lacombe-Harvey, M. E., and Brzezinski, R. (2015). Chitosanases from family 46 of glycoside hydrolases: from proteins to phenotypes. Mar. Drugs 13, 6566-6587. doi: 10.3390/md13116566

Wang, D. W., Li, S. J., Tan, X. Y., Wang, J. H., Hu, Y., Tan, Z., et al. (2021a). Engineering of stepwise-targeting chitosan oligosaccharide conjugate for the treatment of acute kidney injury. Carbohyd. Polym. 256:117556. doi: 10.1016/ j.carbpol.2020.117556

Wang, Y., Li, D., Liu, M., Xia, C., Fan, Q., Li, X., et al. (2021b). Preparation of active chitooligosaccharides with a novel chitosanase AqCoA and their application in fungal disease protection. J. Agr. Food Chem. 11, 3351-3361. doi: 10.1021/acs. jafc.0c07802

Wang, W., Guo, N., Huang, W. C., Zhang, Z. H., and Mao, X. Z. (2018). Immobilization of chitosanases onto magnetic nanoparticles to enhance enzyme performance. Catalysts 8:401. doi: 10.3390/catal8090401

Yan, Y. Q., Tang, J. T., Yuan, Q. F., Gu, Q. N., Liu, H., Huang, J. B., et al. (2020). ChCDC25 regulates infection-related morphogenesis and pathogenicity of the crucifer anthracnose fungus Colletotrichum higginsianum. Front. Microbiol. 11:763. doi: $10.3389 /$ fmicb. 2020.00763 
Yang, G. S., Sun, H. H., Cao, R., Liu, Q., and Mao, X. Z. (2020). Characterization of a novel glycoside hydrolase family 46 chitosanase, Csn-BAC, from Bacillus sp. MD-5. Int. J. Biol. Macromol. 146, 518-523. doi: 10.1016/j.ijbiomac.2020.01.031

Yang, Y., Zheng, Z., Xiao, Y. F., Zhang, J. J., Zhou, Y., Li, X., et al. (2019). Cloning and characterization of a cold-adapted chitosanase from marine bacterium Bacillus sp. BY01. Molecules 24:3915. doi: 10.3390/Molecules24213915

Yun, C., Matsuda, H., and Kawamukai, M. (2006). Directed evolution to enhance secretion efficiency and thermostability of chitosanase from Mitsuaria chitosanitabida 3001. Biosci. Biotech. Bioch. 70, 559-563. doi: 10.1271/bbb.70. 559

Zakariassen, H., Aam, B. B., Horn, S. J., Varum, K. M., Sorlie, M., and Eijsink, V. G. H. (2009). Aromatic residues in the catalytic center of chitinase a from Serratia marcescens affect processivity, enzyme activity, and biomass converting efficiency. J. Bio. Chem. 284, 10610-10617. doi: 10.1074/jbc.M900092200

Zhang, Y. L., Tan, H. M., Wei, G. B., Huang, Y. C., Jian, J. C., and Cai, S. H. (2021). The effect of chitosan oligosaccharide as an immune enhancer against Vibrio harveyiin pearl gentian grouper (female Epinephelus fuscoguttatusx male Epinephelus lanceolatus). Aquac. Res. 52, 541-546. doi: 10.1111/are. 14912

Zheng, Q., Meng, X., Cheng, M., Li, Y., Liu, Y., and Chen, X. (2021). Cloning and characterization of a new chitosanase from a deep-sea Bacterium
Serratia sp. QD07. Front. Microbiol. 12:619731. doi: 10.3389/fmicb.2021. 619731

Zhou, J. L., Liu, X. B., Yuan, F., Deng, B., and Yu, X. B. (2020). Biocatalysis of heterogenously-expressed chitosanase for the preparation of desirable chitosan oligosaccharides applied against phytopathogenic fungi. Acs Sustain. Chem. Eng. 8, 4781-4791. doi: 10.1021/acssuschemeng.9b07288

Zhou, Z. P., Zhao, S. Z., Wang, S. Q., Li, X. M., Su, L., Ma, Y. H., et al. (2015). Extracellular overexpression of chitosanase from Bacillus sp. TS in Escherichia coli. Appl. Biochem. Biotech. 175, 3271-3286. doi: $10.1007 / \mathrm{s} 12010-015-1494-5$

Conflict of Interest: The authors declare that the research was conducted in the absence of any commercial or financial relationships that could be construed as a potential conflict of interest.

Copyright (c) 2021 Cui, Yang, Lu and Shen. This is an open-access article distributed under the terms of the Creative Commons Attribution License (CC BY). The use, distribution or reproduction in other forums is permitted, provided the original author(s) and the copyright owner(s) are credited and that the original publication in this journal is cited, in accordance with accepted academic practice. No use, distribution or reproduction is permitted which does not comply with these terms. 